\title{
Detecting Mechanical Alternans Utilizing Photoplethysmography
}

\author{
Tudor Besleaga ${ }^{1}$, Antonio Canichella $^{2}$, Nicola Toschi ${ }^{2}$, Andreas Demosthenous $^{3}$, Pier D Lambiase ${ }^{4}$, \\ Michele Orini ${ }^{4}$ \\ ${ }^{1}$ Dept. of Medical Physics and Biomedical Engineering, University College London, London, UK \\ ${ }^{2}$ Medical Physics, Department of Biomedicine and Prevention, University of Rome, Rome, Italy \\ ${ }^{3}$ Department of Electronic and Electrical Engineering, University College London, London, UK \\ ${ }^{4}$ Institute of Cardiovascular Science, University College London, London, UK
}

\begin{abstract}
Mechanical alternans (MA) is a biomarker associated with mortality in heart failure patients. Its detection through continuous blood pressure (BP) monitoring is costly and impractical. In this work, we propose the use of photoplethysmography (PPG) as a non-invasive solution for MA detection. Continuous invasive BP and PPG were recorded and analyzed during ventricular pacing in 10 patients. The presence of MA was evaluated in $B P$ and in features characterizing the PPG pulse morphology. Mechanical alternans was defined as an alternation in maximum dP/dt for a duration of 20 consecutive heart beats or more. Mechanical alternans was observed in BP in 5 patients (50\%). The PPG-based MA surrogates showing the highest detection accuracy, were the maximum of the first derivative of the PPG pulse $\left(V^{\prime}{ }_{M}\right)$, and the pulse amplitude (A). Both features allow detection of MA positive patients with 100\% sensitivity and 100\% specificity. The magnitude of MA was correlated between $B P$ and $V{ }_{M}^{\prime} P P G(R=0.92, p<0.001)$ and between $B P$ and A PPG $(R=0.89, p<0.001)$. In conclusion, MA can be accurately detected noninvasively through the PPG.
\end{abstract}

\section{Introduction}

Mechanical alternans (MA; aka blood pressure alternans and pulsus alternans) is a condition whereby blood pressure oscillates on an every other beat basis showing an alternating sequence of strong-weak-strongweak beats. It has been associated with mortality in heart failure patients [1], linked to myocardial ischemia and potentially fatal ventricular arrhythmias [2]. Over time there has been continuing interest in understanding the mechanisms and clinical manifestations of this phenomenon [3].

Despite its potential as a risk marker, the use of MA is seriously limited by the requirement of continuous blood pressure monitoring which is either invasive or performed through cumbersome and expensive devices. In contrast to such continuous blood pressure (BP) monitoring, photoplethysmography (PPG) based devices e.g. pulse oximeters, are non-invasive, cheaper, convenient to use and widely available in hospitals and clinics. The current expansion of wearable devices allowing continuous PPG recordings provides an opportunity for improving platforms for remote care monitoring.

We hypothesise that although non-invasive estimation of BP based on PPG has been proven challenging, the PPG could be used to track fast haemodynamic changes and instabilities. The aim of this study is to explore quantitatively how PPG could be used to detect MA by exploring PPG pulse morphological features as potential surrogates for MA.

\section{Method}

\subsection{Data collection}

A dataset of 10 patients undergoing catheter ablation of ventricular tachycardia (7) and open-heart surgery for coronary artery bypass grafting (3) was analyzed for MA presence. The study was approved by the local research ethics committee and all patients gave informed consent for data collection.

In the Catheterisation Lab invasive aortic blood pressure and PPG were simultaneously measured with sampling frequency equal to $240 \mathrm{~Hz}$, (Mac-Lab System, General Electric), whereas in the cardiac theatre invasive aortic blood pressure and PPG were simultaneously measured with sampling frequencies equal to $100 \mathrm{~Hz}$, (Carescape, General Electric). Ventricular pacing (cycle length equal to $559 \pm 136 \mathrm{~ms}$, range $410-870 \mathrm{~ms}$ ) was established to increase heart rate and induce MA.

Both the PPG and BP raw signals were extracted from the patient monitor, bypassing the auto-gain and display filters. The signals were filtered with a finite impulse 
response (FIR) low pass filter of $30 \mathrm{~Hz}$ cut-off frequency. The filter order was adjusted to the signal sampling frequency. Following a peak detector algorithm, each peak selection was manually verified for consistency and corrected when needed.

\subsection{Definition and Detection of Alternans}

Customized MATLAB 2017b (MathWorks, Inc, Natick, MA) software was developed to detect pulse oscillations in blood pressure and PPG. To exclude ectopic induced oscillations, beats accompanied by changes in cycle length of more than $200 \mathrm{~ms}$ were excluded from the analysis.

For each beat $n$, the maximum of the first derivative of the continuous BP was measured as:

$$
X_{n}=P_{M}^{\prime}(n)=\max _{t \in I_{P I}}\left(\frac{d P(t)}{d t}\right)
$$

where $I_{P I}$ is the interval within the $n_{t h}$ heartbeat, and $t$ is time.

An alternating sequence of $Q$ beats was identified by an uninterrupted succession of beats, showing an alternating pattern (high-low-high-low), satisfying:

$$
\left\{\begin{array}{l}
X_{n} \mid\left(X_{n}>X_{n-1} A N D X_{n}>X_{n+1}\right) \\
O R\left(X_{n}<X_{n-1} A N D X_{n}<X_{n+1}\right)
\end{array}\right\}_{n=Q_{i}}^{Q_{f}}
$$

where $Q_{i}$ and $Q_{f}$ are the initial and final beat numbers of the alternating sequence, and $Q=Q_{f}-Q_{i}+1$. Based on the value of $Q$, MA was detected if $Q \geq 20$.

The beat-to-beat differences were calculated in absolute terms:

$$
\Delta_{a}(n)=\left|X_{n}-X_{n-1}\right|
$$

and in relative terms:

$$
\Delta_{r}(n)=\frac{\Delta_{a}(n)}{\max \left(\left[X_{n}, X_{n-1}\right]\right)} \cdot 100
$$

The magnitude of MA was calculated for each episode of alternans as:

$$
\overline{\Delta_{r}}=\frac{\sum_{n=Q i}^{Q f} \Delta_{r}(n)}{Q}
$$

A relative magnitude of $100 \%$ in $\mathrm{BP}\left(X_{n}=P_{M}^{\prime}(n)\right)$ denotes the presence of total pulsus alternans, i.e. complete suppression of the weak pulse.

Patients that exhibited at least one episode of alternans were classified as MA positive $\left(M A^{+}\right)$. Patients not showing MA were classified as MA negatives $\left(M A^{-}\right)$.

\subsection{PPG Feature Extraction}

Features characterizing the PPG pulse morphology were extracted for every detected heartbeat. Fig. 1 illustrates these features for a single PPG pulse.

Beat-to-beat oscillations in these indices were then analyzed using equation (2), where $X_{n}$ represents the value

\begin{tabular}{|c|c|}
\hline $\mathbf{V}_{\mathbf{M}}$ & lse Peak - the maximum PPG pulse value. \\
\hline $\mathbf{V}^{\prime} \mathbf{M}$ & $\begin{array}{l}\text { First Derivative Peak - the maximum value of the first } \\
\text { derivative of the PPG pulse. }\end{array}$ \\
\hline $\mathbf{V}^{\prime \prime}$ & $\begin{array}{l}\text { Second Derivative Peak - the maximum value of the } \\
\text { second derivative of the PPG pulse. }\end{array}$ \\
\hline $\mathbf{A}$ & de - difference between the $\mathbf{V}_{\mathbf{M}}$ and foot \\
\hline & \\
\hline $\mathbf{a}$ & $\begin{array}{l}\text { Area - The sum of the values for which the PPG pulse } \\
\text { was greater or equal to the value of maximum slope. }\end{array}$ \\
\hline$v$ & Mean - The mean value of PPG over the pulse interval. \\
\hline $\mathbf{I}_{\mathbf{P I}}$ & $\begin{array}{l}\text { Pulse Interval - time interval between } \mathbf{V}{ }^{\prime} \mathbf{M} \text { in two } \\
\text { consecutive pulses. }\end{array}$ \\
\hline
\end{tabular}
of any of the PPG features from Fig. 1.

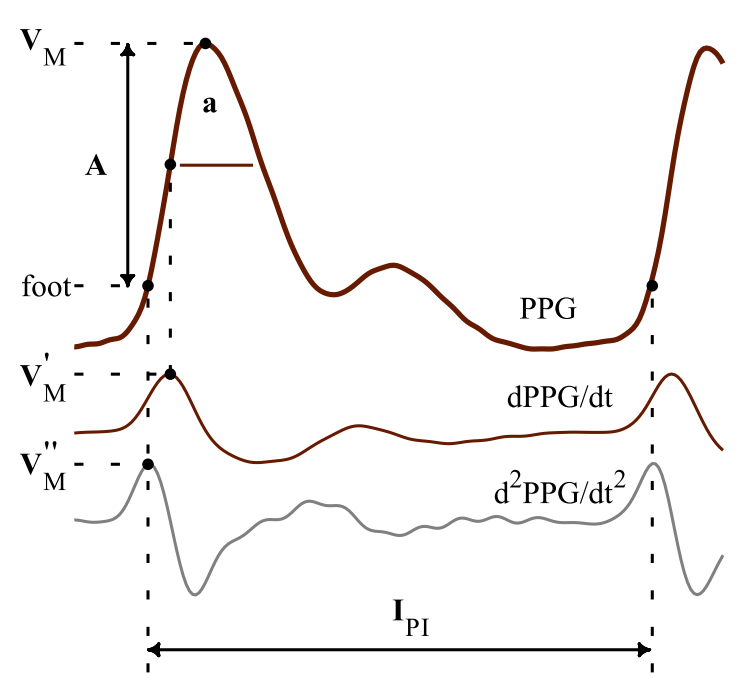

Fig. 1. PPG pulse specific features extracted during a single heartbeat. The $\mathrm{x}$-axis is time and the $\mathrm{y}$-axis represents the magnitude of the signals in arbitrary units.

For each PPG index, the magnitude of MA-surrogates was calculated using equations (3), (4) and (5), where $X_{n}$ represents the values of a pulse specific PPG index.

The presence and magnitude of alternans in PPG was calculated for each index using the same condition $Q \geq 20$ and a threshold $t h$, for which $\overline{\Delta_{r}}>t h$. Receiver operating characteristic (ROC) analysis was performed by incrementally varying the threshold value for the mean relative magnitude $\left(\overline{\Delta_{\mathrm{rPPG}}}\right)$, from $0 \%$ to $60 \%$. For each value, the MA detection accuracy was calculated.

Patients exhibiting MA in PPG indices were classified as true or false positive, based on the presence of MA in BP. Similarly, patients that did not exhibit MA in PPG indices were classified as true or false negative based on the absence of MA in the BP. Sensitivity and specificity were calculated as the true positive rate and true negative rate, respectively. The best magnitude threshold for each PPG index was selected by maximizing the value of Sensitivity multiplied by Specificity. 

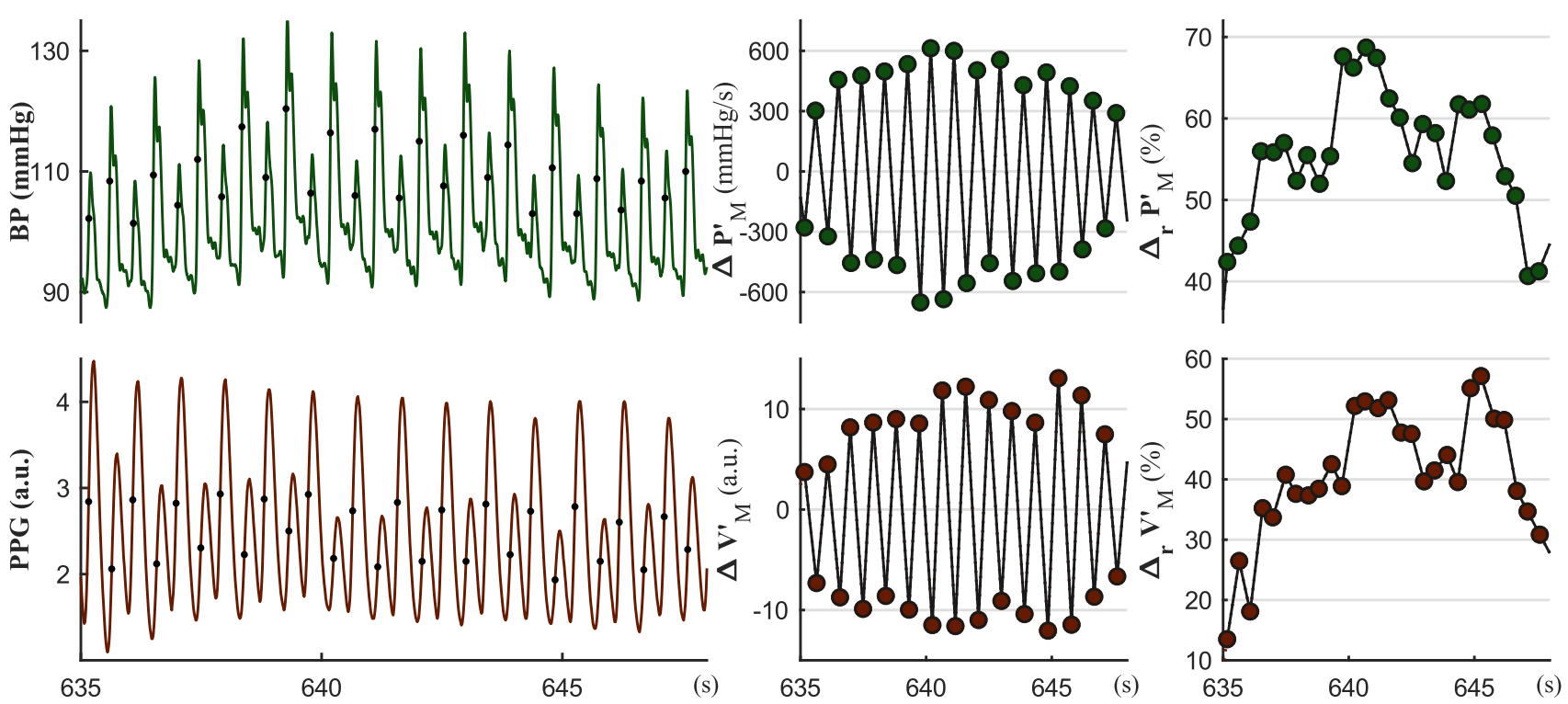

Fig. 2. BP, first row, and PPG, second row, during MA episode. The original signals are represented with markers indicating the maximum slope. $\Delta \mathrm{P}$ ' ${ }_{\mathrm{M}} / \Delta \mathrm{V}$ ' ${ }_{\mathrm{M}}$ represent the beat to beat difference of first derivative maximums. $\Delta_{\mathrm{r}} \mathrm{P}$ ' ${ }_{\mathrm{M}} / \Delta_{\mathrm{r}} \mathrm{V}$ ' ${ }_{\mathrm{M}}$ represent the beat to beat relative difference between the first derivative maximums, see equation (4). The PPG signals were recorded in arbitrary units (Volts).

\section{Results}

Out of 10 patients, 5 exhibited MA. Table 1 summarizes the performance of PPG-based surrogates for detection of MA in patients. Sensitivity, specificity and accuracy relative to the thresholds identified by ROC analysis are shown along with alternans magnitude correlation and the number of detected MA episodes.

In BP, there were 39 detected episodes of MA, of absolute magnitude $224 \mathrm{mmHg} / \mathrm{s}$ ( $\pm 153 \mathrm{mmHg} / \mathrm{s}$ ).

All PPG indices evaluated MA only in relative terms, except for the pulse interval $\left(\mathrm{I}_{\mathrm{PI}}\right)$ which detected 43 episodes of MA of absolute magnitude $72 \mathrm{~ms}( \pm 22 \mathrm{~ms})$.

The pulse derivative $\max \left(V^{\prime}{ }_{M}\right)$ and pulse amplitude $(A)$ detected MA with $100 \%$ sensitivity and $100 \%$ specificity.

Table 1. PPG-based MA surrogates performance

\begin{tabular}{ccccccc}
\hline $\begin{array}{c}\text { PPG } \\
\text { Index }\end{array}$ & $\begin{array}{c}\text { Thresh } \\
(\boldsymbol{\%})\end{array}$ & $\begin{array}{c}\text { Sens } \\
\mathbf{\%}\end{array}$ & $\begin{array}{c}\text { Spec } \\
\mathbf{\%}\end{array}$ & $\begin{array}{c}\text { Accuracy } \\
\mathbf{\%}\end{array}$ & $\begin{array}{c}\text { MA } \\
\text { ep }\end{array}$ & $\begin{array}{c}\text { MA } \\
\text { Corr }\end{array}$ \\
\hline $\mathbf{V}_{M}$ & $(0-10)$ & 100 & 100 & 100 & 41 & $\mathbf{0 . 9 2}$ \\
$\mathbf{A}$ & $(0-16)$ & 100 & 100 & 100 & 46 & $\mathbf{0 . 8 9}$ \\
$\mathbf{a}$ & $(23-29)$ & 100 & 90 & 93 & 36 & $\mathbf{0 . 7 6}$ \\
$\mathbf{V}^{\prime}{ }_{M}$ & $(0-18)$ & 80 & 100 & 93 & 37 & $\mathbf{0 . 9 8}$ \\
$\mathbf{V} \mathbf{M}$ & $(0-27)$ & 80 & 100 & 93 & 37 & $\mathbf{0 . 6 3}$ \\
$\mathbf{I P I}$ & $(0-3)$ & 80 & 90 & 87 & 43 & $\mathbf{0 . 9 3}$ \\
$\overline{\mathbf{V}}$ & $(0-15)$ & 60 & 100 & 87 & 38 & - \\
\hline \hline
\end{tabular}

Thresh - Threshold interval, Sens - sensitivity, Spec specificity, MA ep - number of MA episodes detected in the dataset. MA Corr - Pearson Correlation between MA in BP and in PPG (when detected in 4 patients or more)
The correlation between the magnitude of MA in BP and the magnitude of PPG-based MA was high for most features. The highest correlation was for the second derivative max, but detection of $M A^{+}$patients had low sensitivity; only 4 out of 5 patients were detected. Index $V_{M}^{\prime}$ outperformed the other indices, showing a Pearson correlation with BP MA of 0.92 , and a similar number of detected MA episodes: 41 vs 39. A scatter plot of the relative magnitudes of alternans in $\mathrm{BP}$ and $V^{\prime}{ }_{M} \mathrm{PPG}$ in each patient is presented in Fig. 3.

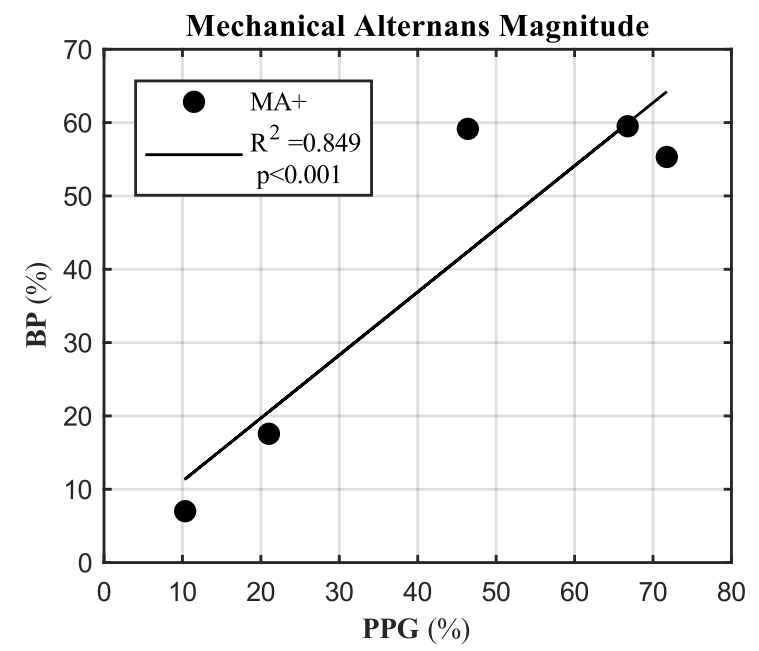

Fig. 3. Correlation between MA magnitude in BP and in PPG $V$ ' ${ }_{M}$. Each point represents a patient with alternans. The coordinates represent the mean relative magnitude of all the alternans episodes detected in a patient. The line fitting is done for all the patients with MA, Pearson Correlation $\mathrm{R}=0.92 ; \mathrm{R}^{2}=$ $0.849 ; \mathrm{p}<0.001$. 


\section{Discussion and Conclusion}

Our results demonstrate that pacing induced MA could be accurately detected non-invasively in patients utilizing PPG. Furthermore, the magnitude of MA can be accurately quantified from the PPG waveform under motion free conditions. The presence of MA has been sufficient to identify patients with cardiac risk in heart failure populations [1], [4]. Mechanical alternans may represent a novel marker for cardiac risk, and its detection through PPG could be a valuable tool in monitoring cardiac patients in a world with an aging population. The ability to detect this marker with existing, widely available medical equipment [5], [6], opens avenues for further clinical investigations into its prognostic utility as a population based risk-stratifier. This optimization could enable more widespread use of PPG data both in the hospital setting and for home monitoring to enable treatment and risk stratification in community care.

Our hypothesis was that PPG could be used to track oscillations in the blood pressure, and therefore, MA could be detected via PPG regardless of the underlying mechanisms, due to the intrinsic mechanical link between arterial pressure and flow [7]. Previous studies have shown that MA can be observed in both ventricular blood pressure [2] and in the peripheral arterial blood pressure [8], with significant increase in magnitude compared to left ventricle alternans. Due to peripheral blood pressure augmentation and the PPG's ability to detect changes in peripheral blood flow [6], it was assumed that alternans could be optically detected at peripheral sites such as the finger.

During MA, small variations appeared in the PPG pulse interval, although the cycle length was constant. Similar temporal variations have been reported in the pulse arrival time [9] during steady state pacing. In sinus rhythm, when the pulse interval can change considerably, the pulse arrival time is potentially another non-invasive marker of MA.

The presence of alternans was investigated independently in each PPG pulse feature, in order to find a direct surrogate for MA that is easy to measure and implement. Our results support the hypothesis that beat to beat oscillations in BP manifest in the PPG waveform, but studies with larger populations are needed to further investigate clinical implications. With the expansion of wearable devices and $\mathrm{m}$-health, one could speculate that MA could be integrated into an adaptive multi-variate riskprediction model based on data recorded through a PPG sensor continuously.

This work demonstrates that pacing-induced MA can be detected from the PPG signal without resource intensive algorithms. This finding has implications for screening of MA and quantifying heart failure progression.

\section{Acknowledgements}

This work was funded by University College of London and Integrated Technologies Ltd (www.itlmedical.com). PD Lambiase acknowledges support from the UCLH Biomedicine NIHR. M Orini was supported by an IEF 2013 Marie Curie Fellowship.

\section{References}

[1] R. Kim et al., "Mechanical alternans is associated with mortality in acute hospitalized heart failure: prospective mechanical alternans study (MAS)," Circ. Arrhythmia Electrophysiol., vol. 7, no. 2, pp. 259-266, Apr. 2014.

[2] B. Surawicz and C. Fisch, "Cardiac alternans: diverse mechanisms and clinical manifestations," J. Am. Coll. Cardiol., vol. 20, no. 2, pp. 483-499, 1992.

[3] D. E. Euler, "Cardiac alternans-mechanisms and pathophysiological significance," Cardiovasc Res, vol. 42, pp. 583-90, 1999.

[4] A. Hirashiki et al., "Prognostic value of pacing-induced mechanical alternans in patients with mild-to-moderate idiopathic dilated cardiomyopathy in sinus rhythm," $J$. Am. Coll. Cardiol., vol. 47, no. 7, pp. 1382-1389, Apr. 2006.

[5] A. A. Alian and K. H. Shelley, "Photoplethysmography," Best Pract. Res. Clin. Anaesthesiol., vol. 28, no. 4, pp. 395-406, 2014.

[6] J. Allen, "Photoplethysmography and its application in clinical physiological measurement," Physiol. Meas., vol. 28, no. 3, pp. R1-R39, 2007.

[7] W. W. Nichols, M. F. O'Rourke, C. Vlachopolus, and D. A. McDonald, McDonald's blood flow in arteries: theoretical, experimental and clinical principles, 6 th ed. London: London : Hodder Arnold, 2011.

[8] R. J. Selvaraj, A. Suszko, A. Subramanian, S. Mak, R. Wainstein, and V. S. Chauhan, "Microscopic systolic pressure alternans in human cardiomyopathy: noninvasive evaluation of a novel risk marker and correlation with microvolt T-wave alternans," Hear. Rhythm, vol. 8, no. 2, pp. 236-243, Feb. 2011.

[9] S. van Duijvenboden, N. Child, B. Hanson, J. Gill, P. Taggart, and M. Orini, "Pulse arrival time accurately detects pacing-induced mechanical alternans," Comput. Cardiol., vol. 44, pp. 2-5, 2017.

Address for correspondence.

Tudor Besleaga

Rayne Institute, 5 University St, Fitzrovia, London WC1E 6JF, UK

tudor.besleaga.09@ucl.ac.uk. 